RESEARCH ARTICLE

\title{
Simultaneous observation of ultrafast electron and proton beams in TNSA
}

\author{
Fabrizio Bisesto $^{\circledR 1}$, Mario Galletti ${ }^{\circledR 2,3}$, Maria Pia Anania ${ }^{\circledR 1}$, Gemma Costa ${ }^{\circledR 1}$, Massimo Ferrario ${ }^{\circledR 1}$, \\ Riccardo Pompili $^{\circledR 1}$, Arie Zigler ${ }^{1,4}$, Fabrizio Consoli ${ }^{\circledR 5}$, Mattia Cipriani ${ }^{\circledR 5}$, Martina Salvadori ${ }^{5,6,7}$, \\ and Claudio Verona ${ }^{108}$ \\ ${ }^{1}$ INFN-LNF, Via Enrico Fermi 54, 00044 Frascati, Italy \\ ${ }^{2}$ Central Laser Facility, Science and Technology Facilities Council, Rutherford Appleton Laboratory, Harwell Science and Innovation \\ Campus, Didcot OX11 OQX, England \\ ${ }^{3}$ GoLP Instituto de Plasmas e Fusão Nuclear, Instituto Superior Tecnico, Universidade de Lisboa, Av. Rovisco Pais, 1049-001 Lisbon, \\ Portugal \\ ${ }^{4}$ Racah Institute of Physics, Hebrew University, 91904 Jerusalem, Israel \\ ${ }^{5}$ ENEA Fusion and Technologies for Nuclear Safety and Security Department, C.R. Frascati, Via E. Fermi 45, 00044 Frascati, Italy \\ ${ }^{6}$ Universita' di Roma La Sapienza, Piazzale Aldo Moro 5, 00185 Roma, Italy \\ ${ }^{7}$ INRS-EMT, Varennes, Québec, Canada \\ ${ }^{8}$ University of Rome "Tor Vergata", Industrial Engineering Department, Via Cracovia 50, 00133 Roma, Italy \\ (Received 10 January 2020; revised 13 March 2020; accepted 1 April 2020)
}

\begin{abstract}
The interaction of ultra-intense high-power lasers with solid-state targets has been largely studied for the past 20 years as a future compact proton and ion source. Indeed, the huge potential established on the target surface by the escaping electrons provides accelerating gradients of $\mathrm{TV} / \mathrm{m}$. This process, called target normal sheath acceleration, involves a large number of phenomena and is very difficult to study because of the picosecond scale dynamics. At the SPARC_LAB Test Facility, the high-power laser FLAME is employed in experiments with solid targets, aiming to study possible correlations between ballistic fast electrons and accelerated protons. In detail, we have installed in the interaction chamber two different diagnostics, each one devoted to characterizing one beam. The first relies on electro-optic sampling, and it has been adopted to completely characterize the ultrafast electron components. On the other hand, a time-of-flight detector, based on chemical-vapour-deposited diamond, has allowed us to retrieve the proton energy spectrum. In this work, we report preliminary studies about simultaneous temporal resolved measurements of both the first forerunner escaping electrons and the accelerated protons for different laser parameters.
\end{abstract}

Keywords: electro-optic sampling diagnostics; high-power laser; laser-plasma interaction; time-of-flight diagnostics; target normal sheath acceleration; ultrashort high-intensity laser pulses

\section{Introduction}

The invention of chirped pulse amplification ${ }^{[1]}$ has provided a huge boost to laser technology, allowing the development of ultrashort lasers. In less than 20 years, TW $\left(10^{12} \mathrm{~W}\right)$ and PW $\left(10^{15} \mathrm{~W}\right)$ class systems have been built around the world ${ }^{[2]}$, becoming extremely useful tools to study the interaction with plasma.

In particular, the use of intensities $I_{L}>10^{18} \mathrm{~W} / \mathrm{cm}^{2}$ permits us to study interactions in the relativistic regime with solid-state matter opening the way to deliver completely

Correspondence to: F. Bisesto, INFN-LNF, Via Enrico Fermi 54, 00044 Frascati, Italy. Email: fabrizio.giuseppe.bisesto@lnf.infn.it new and very compact proton accelerators at the millimetre scale $^{[3-7]}$.

The ion/proton production and acceleration processes are extremely connected to the electron population directly accelerated by the laser in the early stage of the so-called target normal sheath acceleration (TNSA) phenomenon. The electron energy spectrum is distributed following a Maxwell distribution with a characteristic energy

$$
T_{\text {hot }}=E_{0} \times\left(\sqrt{1+a_{0}^{2}}-1\right) \text {, }
$$

where $a_{0}$ is the normalized laser potential. 
These hot electrons cross the target and leave an unbalanced positive charge on it, establishing a quasi-static potential. While most of them are stopped in the vicinity of the back surface, within a distance of the Debye length ${ }^{[8]}$, only the fastest ones, constituting a small fraction of the entire population, can completely escape from the potential. The latter, in turn, is responsible for ion acceleration ${ }^{[9,10]}$. Studying the fast component of the electron population, hereinafter called ultrafast electrons, can reveal an interesting aspect of the whole process and help to optimize the proton acceleration.

At the SPARC LAB Test Facility ${ }^{[11]}$, ultrafast electrooptic sampling (EOS) diagnostics ${ }^{[12,13]}$ have been installed in the FLAME laser ${ }^{[14]}$ target area. This allows us to perform temporally resolved measurements on fast electrons with about 100 fs resolution ${ }^{[15,16]}$. In a previous work, we have shown how this diagnostic tool can investigate electron beam properties, in terms of charge, energy and duration, while changing the target geometry ${ }^{[17]}$. Moreover, we have also employed our EOS probing line to study the evolution of electric fields emitted by high-intensity lasers $\left(I_{L}>\right.$ $10^{18} \mathrm{~W} / \mathrm{cm}^{2}$ ) impinging on solid targets ${ }^{[18,19]}$.

In addiction, we added a time-of-flight (TOF) diamond detector to the pre-existing experimental setup ${ }^{[20]}$, employed as proton energy spectrum diagnostics ${ }^{[21-24]}$. In the present work, we report preliminary studies on simultaneous time resolved measurements about ultrafast electron and proton populations by varying laser parameters.

\section{Experimental setup}

The experiment has been carried out at the SPARC_LAB Test Facility ${ }^{[11]}$ by exploiting the high-power laser FLAME $^{[14]}$, delivering routinely down to $25 \mathrm{fs}$ and up to $4 \mathrm{~J}$ pulses at $10 \mathrm{~Hz}$ on the target. After optical compression, the laser beam is focused by means of an $f / 10$ off-axis parabolic mirror, reaching a final spot size down to $15 \mu \mathrm{m}\left(1 / e^{2}\right.$ radius). Thus, a peak intensity up to $\sim 3 \times 10^{19} \mathrm{~W} / \mathrm{cm}^{2}$ can be achieved in the target area, held in a high-vacuum environment $\left(10^{-6} \mathrm{mbar}\right)$, corresponding to a normalized intensity $a_{0} \approx 3$. Moreover, the high temporal contrast $\left(10^{-8}\right.$ at $10 \mathrm{ps}$ and $10^{-10}$ at $>100 \mathrm{ps}$ ) makes our system suitable for plasma acceleration studies.

A small portion of the main beam is split and used as a completely temporal jitter-free probe laser line. The two laser beams are synchronized at the fs level in the interaction point by means of an autocorrelator, consisting of an $\alpha$-BBO crystal and a delay line. The latter is also used to perform a temporal scan of the interaction in a range of $500 \mathrm{ps}$.

The experimental setup is shown in Figure 1. The interaction with solid matter consists in aluminium foil targets hit

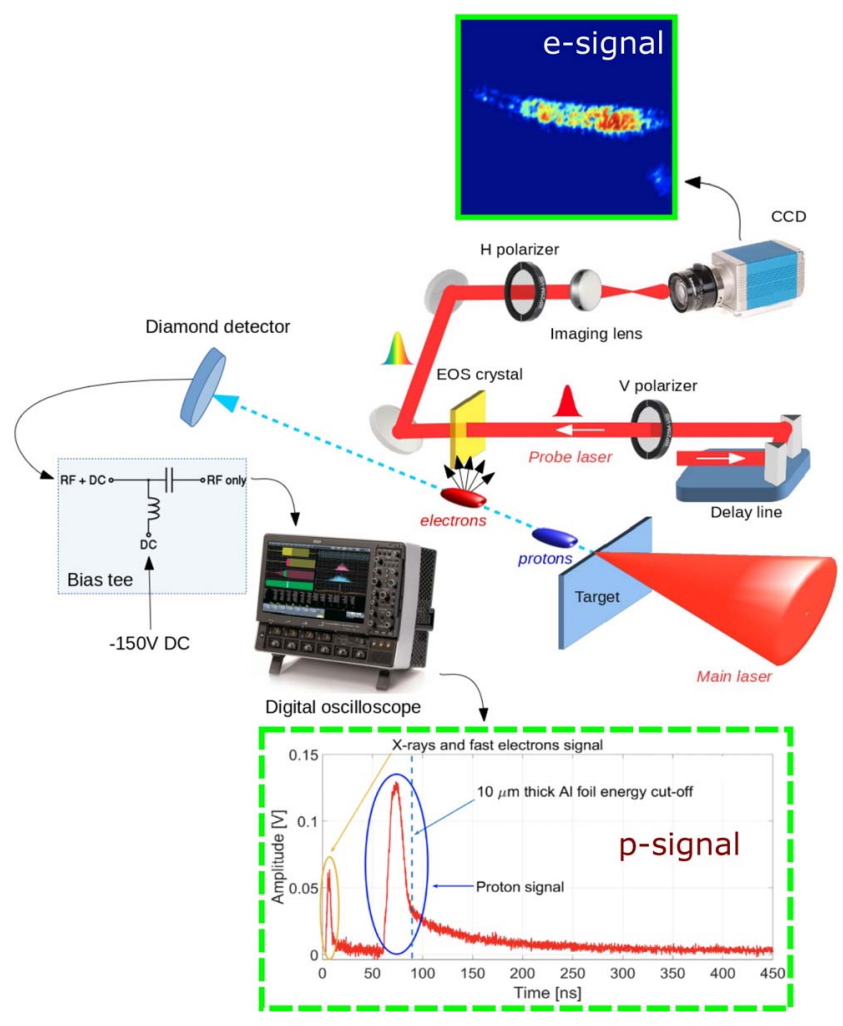

Figure 1. Experimental setup. The FLAME laser is sent to an aluminium target. The charged particles emitted during this interaction are revealed by two single-shot time resolved measurements: electro-optic sampling diagnostics, able to measure the electric field carried by relativistic fast electrons, and a time-of-flight diamond detector, used to measure the temporal distribution of protons arriving at it and to retrieve their energy spectra $^{[20]}$.

by the FLAME laser pulses. The target holder was able to host $1 \mathrm{~cm} \times 5 \mathrm{~cm}$ (height by width) aluminium foils. The holder was constructed by two aluminium plates containing 100 holes, $3 \mathrm{~mm}$ in diameter, corresponding to the available number of shots per target, placed at a $0^{\circ}$ angle with respect to the laser. The ultrafast electrons, able to escape the target, are detected by an electro-optic ZnTe crystal. Here, their electric field can induce a local birefringence that, in turn, can be probed by a laser beam. In particular, in the spatial encoding scheme ${ }^{[25]}$, the electrons' longitudinal distribution can be mapped in the probe laser transverse profile. Then, by means of a polarization decoding line, the longitudinal charge distribution can be measured. Due to the setup geometry and probe laser size, a temporal window of $8 \mathrm{ps}$ with $100 \mathrm{fs}$ resolution is achieved.

The temporal structure of the positively charged beam has been measured by means of an electromagnetic-pulse-free TOF diamond detector ${ }^{[26,27]}$, placed $1.05 \mathrm{~m}$ downstream of the target at $0^{\circ}$ with respect to the laser direction. This tool allowed us to retrieve the proton energy spectrum for each $\operatorname{shot}^{[20]}$. 
a)

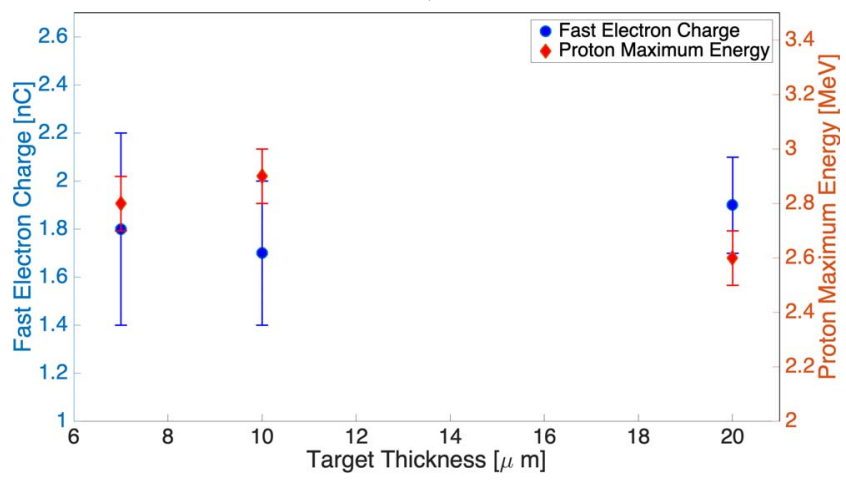

b)

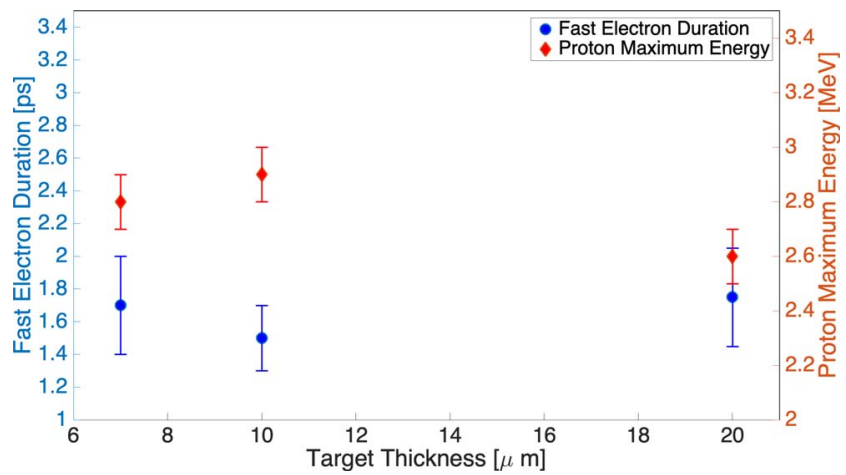

Figure 2. Simultaneous detection of the (a) ultrafast electron charge, (b) temporal length, and maximum proton energy for different available target thicknesses.

\section{Experimental results and discussion}

We have performed simultaneous measurements on ultrafast electron charge and temporal length, and proton energy by changing the laser temporal length from 30 to $300 \mathrm{fs}$ (full width at half maximum (FWHM)) and the focal spot size from 30 to $120 \mu \mathrm{m}\left(1 / e^{2}\right.$ diameter $)$.

The laser energy on the target was kept constant at $2 \mathrm{~J}$.

All the experimental values are reported with their own statistical error, since more shots have been collected for each parameter set.

Initially, we tried to determine our best thickness value among the available ones, i.e., $7 \mu \mathrm{m}, 10 \mu \mathrm{m}$ and $20 \mu \mathrm{m}$, optimizing the proton energy as seen in previous works ${ }^{[28]}$. Thin targets $(\sim 1 \mu \mathrm{m})$ seem to be preferable, but they can be massively perturbed by the nanosecond-long laser pedestal coming before the main pulse, able even to destroy the target. Unfortunately, in the explored range, both the charge and the temporal duration of ultrafast electrons and the proton maximum energy are constant within the statistical error.

Figure 2 shows the values measured for different target thicknesses. We decided to adopt, for this experimental campaign, the $10 \mu \mathrm{m}$ thickness due to the fact that it has yielded a slightly better result in terms of maximum proton a)

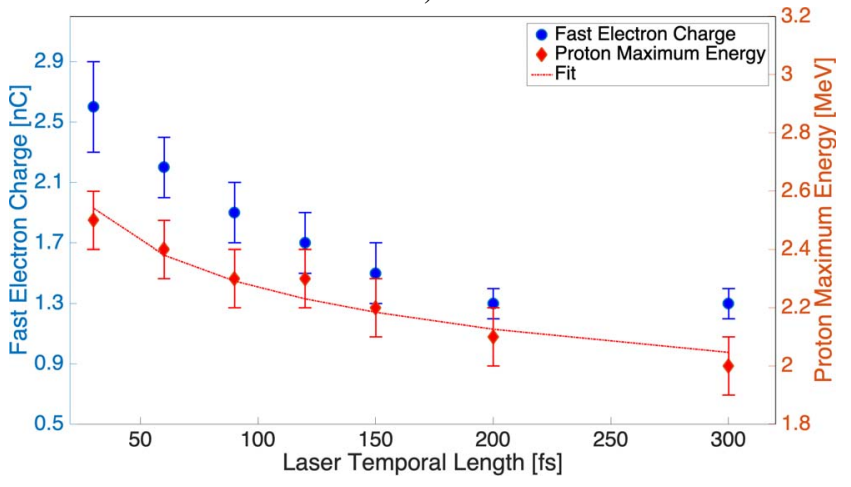

b)

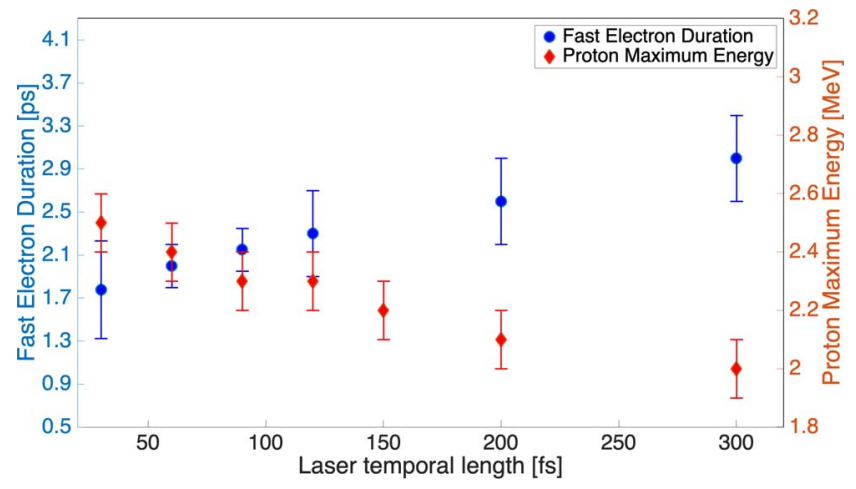

Figure 3. Simultaneous detection of the (a) ultrafast electron charge, (b) temporal length, and maximum proton energy for different laser durations.

energy, as measured in the subtended solid angle of the TOF diagnostic tool. Although a well-known scaling law ${ }^{[28]}$ predicts a maximum proton energy of the order of tens of $\mathrm{MeV}$, energies of few $\mathrm{MeV}$ have been experimentally retrieved as shown in Refs. [29, 30], for our experimental conditions in terms of target thickness and laser pulse duration. However, more measurements related to different target thicknesses and geometries should be performed to try to enhance the maximum proton energy and to optimize the spectra. On the other hand, concerning the ultrafast electron population, while the charge remains constant by varying the target thickness, we measured the shortest temporal length for the $10 \mu \mathrm{m}$ case.

Once the best performing target was implemented in the setup, the experimental campaign was focused on the behaviour of the electron and the proton varying the laser parameters.

For both the laser spot size and temporal duration scaling, the proton measured energy has been fitted with a power law, namely,

$$
F(x)=\alpha x^{\beta},
$$

where $\alpha$ and $\beta$ are free parameters of the fit.

Figure 3 reports the experimental data regarding the electron beam charge and the temporal duration in comparison 
a)

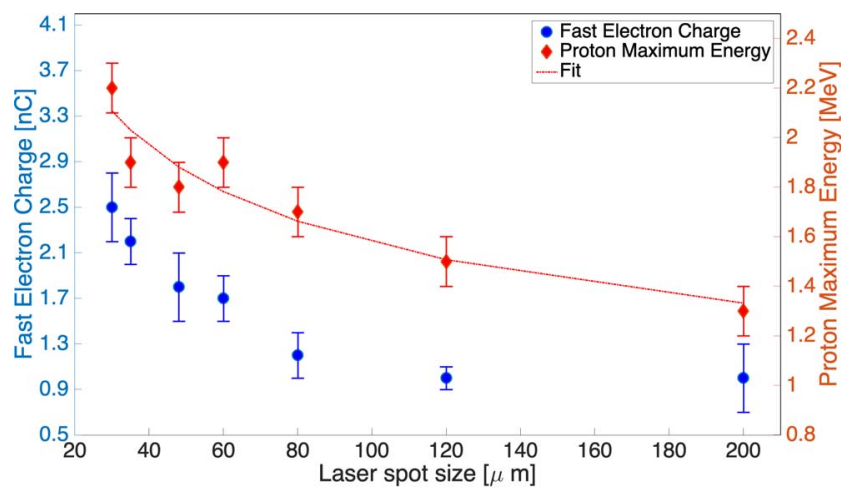

b)

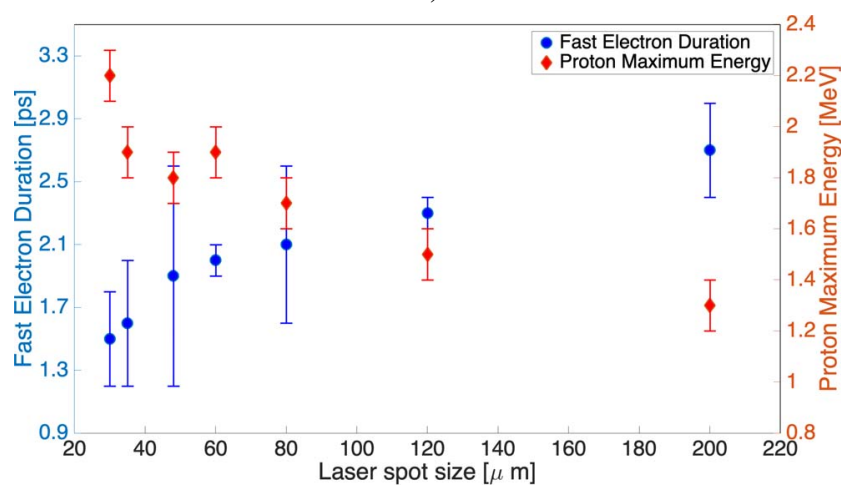

Figure 4. Simultaneous detection of the (a) ultrafast electron charge, (b) temporal length, and maximum proton energy for different spot sizes.

with the proton maximum energy for different laser temporal durations. From the experimental data regarding the proton beam, the following relation is found:

$$
E_{p}^{\max }[\mathrm{MeV}]=(3.5 \pm 0.4) \tau_{L}[\mathrm{fs}]^{(-0.09 \pm 0.03)} .
$$

The maximum proton energy is almost constant, as noticed in Ref. [30] using the same time duration range, target thickness, etc., but for lower laser energy. Meanwhile, the extracted fast electron charge decreases exponentially with laser temporal length and the temporal duration increases linearly.

This can be explained starting from the characteristic hot electron temperature $T_{h}$, depending on the laser normalized potential vector $a_{0} \propto I_{L}^{0.5}$. Therefore, the lower the laser intensity, the weaker the fast electrons, leading to a temporally stretched bunch. Moreover, the number of particles able to escape the target potential and detected by our EOS diagnostics is smaller.

Figure 4 shows the experimental data regarding the electron beam charge and the temporal duration in comparison with the proton maximum energy for different laser spot sizes.
We found for the maximum proton energy as a function of the laser spot size $r_{L}$ (FWHM)

$$
E_{p}^{\max }[\mathrm{MeV}]=(4.8 \pm 0.2) r_{L}[\mu \mathrm{m}]^{(-0.25 \pm 0.09)} .
$$

Contrary to the laser temporal length scaling, in this scenario, both the proton energy and the number of energetic electrons exponentially decrease, as the laser on the target becomes wider, with the intensity diminishing accordingly. On the other hand, the electron beam temporal duration behaviour is still linear.

Furthermore, rewriting Equation (3) as

$$
E_{p}^{\max }[\mathrm{MeV}]=(4.8 \pm 0.2) r_{L}[\mu \mathrm{m}]^{2 \cdot(-0.125 \pm 0.045)}
$$

and considering the laser intensity $\left(I_{L}=E_{L} /\left(\pi r_{L}^{2} \tau_{L}\right)\right)$ dependence on the spot size squared and the pulse duration, from Equations (2) and (3), we can infer $E_{p}^{\max }$ as a function of the laser intensity,

$$
E_{p}^{\max } \propto I_{L}^{(-0.1 \pm 0.04)},
$$

keeping the laser energy constant. Such a relation is valid for a laser pulse with $\tau_{L}=30-300 \mathrm{fs}, w_{0}=15-60 \mu \mathrm{m}$, at a constant laser energy equal to $2 \mathrm{~J}$, corresponding to a laser intensity range $I_{L}=0.1-2 \times 10^{19} \mathrm{~W} / \mathrm{cm}^{2}$, impinging on an aluminium target of $10 \mu \mathrm{m}$ thickness.

\section{Comments}

The two different scenarios explored in this experimental campaign gave us important feedback on the TNSA process.

- The ultrafast electron population, different from the well-studied hot electron population mentioned in the literature, strictly depends on the laser intensity. In both scenarios, stretching the pulse and widening the focal spot, we retrieved the same behaviour, i.e., exponential decreasing.

- In both scenarios, the ultrafast electron population temporal duration shows the same linear behaviour.

- The proton maximum energy is strictly dependent on the energy of the laser, as reported in the literature. According to our measurements, it seems almost constant when varying the laser duration (Equation (2)), while being more sensitive to the laser spot size (Equation (3)). Finally, combining the two relations, we found that $E_{p}^{\max }$ is weakly dependent on the laser intensity $I_{L}$ (Equation (4)).

- Detecting the ultrafast electron charge can give us a clear hint about the proton maximum energy in the case of the laser energy and spatial extension scanning because of their reported behaviour. 


\section{Conclusions}

In conclusion, a characterization of the emission of fast electrons and protons, occurring in ultra-intense laser and solid matter interactions, has been performed by mainly varying the laser temporal duration and focal spot size, using two different detectors. Indeed, thanks to $100 \mathrm{fs}$ temporal resolution EOS diagnostics, fast electron charge has been measured, while a chemical-vapour-deposited diamond-based TOF detector has provided the proton energy spectra.

We found an optimum thickness of $10 \mu \mathrm{m}$, obtaining the highest proton energy, $\sim 2.9 \pm 0.1 \mathrm{MeV}$, for our experimental conditions, while the fast electron charge was almost constant, $\sim 1.8 \pm 0.4 \mathrm{nC}$. By varying the laser temporal duration in the 30-300 fs range, we observed a constant behaviour in the maximum proton energy, as confirmed in Ref. [30]. In turn, the fast electron charge, detected by our EOS monitor, decreases as the laser becomes longer. Measurements were performed also for different laser intensities by changing the laser spot size on the target. The fast electron charge, as well as the maximum proton energy, decreases with reduction in the laser power density. Our measurements are in agreement with previous works where these scaling laws on the proton maximum energy were studied for femtosecond laser pulses $^{[28,31-33]}$. Concerning the ultrafast electron temporal length, we observed a stretching effect by decreasing the laser pulse intensity. This can be explained as being due to a higher velocity spread in the electron population, which becomes less relativistic since its energy strongly depends on the normalized laser vector potential $a_{0}$.

These results show the potentialities of our simultaneous detection system for both fast electrons and protons emitted as a consequence of the interaction between a highintensity laser pulse and a solid-state target. In particular, it may be employed to better understand the whole TNSA phenomenon and how the fast electron beam can influence the proton acceleration and, at the same time, how it can be used to infer the expected proton spectra.

\section{References}

1. D. Strickland and G. Mourou, Opt. Commun. 56, 219 (1985).

2. C. Danson, D. Hillier, N. Hopps, and D. Neely, High Power Laser Sci. Eng. 3, e3 (2015).

3. R. A. Snavely, M. H. Key, S. P. Hatchett, T. E. Cowan, M. Roth, T. W. Phillips, M. A. Stoyer, E. A. Henry, T. C. Sangster, M. S. Singh, S. C. Wilks, A. MacKinnon, A. Offenberger, D. M. Pennington, K. Yasuike, A. B. Langdon, B. F. Lasinski, J. Johnson, M. D. Perry, and E. M. Campbell, Phys. Rev. Lett. 85, 2945 (2000).

4. S. C. Wilks, A. B. Langdon, T. E. Cowan, M. Roth, M. Singh, S. Hatchett, M. H. Key, D. Pennington, A. MacKinnon, and R. A. Snavely, Phys. Plasmas 8, 542 (2001).

5. M. Roth, T. E. Cowan, M. H. Key, S. P. Hatchett, C. Brown, W. Fountain, J. Johnson, D. M. Pennington, R. A. Snavely, S. C. Wilks, K. Yasuike, H. Ruhl, F. Pegoraro, S. V. Bulanov, E. M. Campbell, M. D. Perry, and H. Powell, Phys. Rev. Lett. 86, 436 (2001).
6. H. Schwoerer, S. Pfotenhauer, O. Jäckel, K.-U. Amthor, B. Liesfeld, W. Ziegler, R. Sauerbrey, K. W. D. Ledingham, and T. Esirkepov, Nature 439, 445 (2006).

7. T. Bartal, M. E. Foord, C. Bellei, M. H. Key, K. A. Flippo, S. A. Gaillard, D. T. Offermann, P. K. Patel, L. C. Jarrott, D. P. Higginson, M. Roth, A. Otten, D. Kraus, R. B. Stephens, H. S. McLean, E. M. Giraldez, M. S. Wei, D. C. Gautier, and F. N. Beg, Nature Phys. 8, 139 (2012).

8. J. Badziak, S. Głowacz, S. Jabłoński, P. Parys, J. Wołowski, H. Hora, J. Krása, L. Láska, and K. Rohlena, Plasma Phys. Control. Fusion 46, B541 (2004).

9. A. G. Krygier, D. W. Schumacher, and R. R. Freeman, Phys. Plasmas 21, 023112 (2014).

10. A. Macchi, M. Borghesi, and M. Passoni, Rev. Mod. Phys. 85, 751 (2013).

11. M. Ferrario, D. Alesini, M. Anania, A. Bacci, M. Bellaveglia, O. Bogdanov, R. Boni, M. Castellano, E. Chiadroni, A. Cianchi, S. B. Dabagov, C. De Martinis, D. Di Giovenale, G. Di Pirro, U. Dosselli, A. Drago, A. Esposito, R. Faccini, A. Gallo, M. Gambaccini, C. Gatti, G. Gatti, A. Ghigo, D. Giulietti, A. Ligidov, P. Londrillo, S. Lupi, A. Mostacci, E. Pace, L. Palumbo, V. Petrillo, R. Pompili, A. R. Rossi, L. Serafini, B. Spataro, P. Tomassini, G. Turchetti, C. Vaccarezza, F. Villa, G. Dattoli, E. Di Palma, L. Giannessi, A. Petralia, C. Ronsivalle, I. Spassovsky, V. Surrenti, L. Gizzi, L. Labate, T. Levato, and J. V. Rau, Nucl. Instrum. Methods Phys. Res. B 309, 183 (2013).

12. F. Bisesto, M. Anania, P. Maria, M. Botton, E. Chiadroni, A. Cianchi, A. Curcio, M. Ferrario, M. Galletti, R. Pompili, E. Schleifer, and A. Zigler, Quantum Beam Sci. 1, 13 (2017).

13. F. G. Bisesto, M. P. Anania, M. Botton, M. Castellano, E. Chiadroni, A. Cianchi, A. Curcio, M. Ferrario, M. Galletti, Z. Henis, R. Pompili, E. Schleifer, V. Shpakov, and A. Zigler, Nucl. Instrum. Methods Phys. Res. A 909, 364 (2018).

14. F. G. Bisesto, M. P. Anania, M. Bellaveglia, E. Chiadroni, A. Cianchi, G. Costa, A. Curcio, D. Di Giovenale, G. Di Pirro, M. Ferrario, M. Galletti, Z. Henis, R. Pompili, E. Schleifer, and A. Zigler, Nucl. Instrum. Methods Phys. Res. A 909, 452 (2018).

15. R. Pompili, M. P. Anania, F. Bisesto, M. Botton, M. Castellano, E. Chiadroni, A. Cianchi, A. Curcio, M. Ferrario, M. Galletti, Z. Henis, M. Petrarca, E. Schleifer, and A. Zigler, Opt. Express 24, 29512 (2016).

16. M. Galletti, F. G. Bisesto, M. P. Anania, M. Ferrario, R. Pompili, A. Poyé, V. Tikhonchuk, and A. Zigler, Appl. Phys. Lett. 116, 064102 (2020).

17. R. Pompili, M. P. Anania, F. Bisesto, M. Botton, M. Castellano, E. Chiadroni, A. Cianchi, A. Curcio, M. Ferrario, M. Galletti, Z. Henis, M. Petrarca, E. Schleifer, and A. Zigler, Sci. Rep. 6, 35000 (2016).

18. R. Pompili, M. P. Anania, F. Bisesto, M. Botton, E. Chiadroni, A. Cianchi, A. Curcio, M. Ferrario, M. Galletti, Z. Henis, M. Petrarca, E. Schleifer, and A. Zigler, Sci. Rep. 8, 3243 (2018).

19. F. G. Bisesto, M. P. Anania, M. Botton, E. Chiadroni, A. Cianchi, A. Curcio, M. Ferrario, M. Galletti, Z. Henis, R. Pompili, E. Schleifer, and A. Zigler, Nucl. Instrum. Methods Phys. Res. A 909, 398 (2018).

20. F. Bisesto, M. Galletti, M. P. Anania, M. Ferrario, R. Pompili, M. Botton, A. Zigler, F. Consoli, M. Salvadori, P. Andreoli, and C. Verona, High Power Laser Sci. Eng. 7, e53 (2019).

21. D. Margarone, J. Krasa, L. Giuffrida, A. Picciotto, L. Torrisi, T. Nowak, P. Musumeci, A. Velyhan, J. Prokuupek, L. Laska, T. Mocek, J. Ullschmied, and B. Rus, J. Appl. Phys. 109, 103302 (2011). 
22. S. Busold, D. Schumacher, O. Deppert, C. Brabetz, S. Frydrych, F. Kroll, M. Joost, H. Al-Omari, A. Blažević, B. Zielbauer, I. Hofmann, V. Bagnoud, T. E. Cowan, and M. Roth, Phys. Rev. Spec. Top. Accel. Beams 16, 101302 (2013).

23. L. A. Gizzi, D. Giove, C. Altana, F. Brandi, P. Cirrone, G. Cristoforetti, A. Fazzi, P. Ferrara, L. Fulgentini, P. Koester, L. Labate, G. Lanzalone, P. Londrillo, D. Mascali, A. Muoio, D. Palla, F. Schillaci, S. Sinigardi, S. Tudisco, and G. Turchetti, Appl. Sci. 7, 984 (2017).

24. F. Bisesto, M. Galletti, M. P. Anania, M. Ferrario, R. Pompili, M. Botton, E. Schleifer, and A. Zigler, High Power Laser Sci. Eng. 7, e56 (2019).

25. A. L. Cavalieri, Electro-Optic Characterization of Femtosecond Electron Bunches (University of Michigan, 2005).

26. R. De Angelis, F. Consoli, C. Verona, G. Di Giorgio, P. Andreoli, G. Cristofari, M. Cipriani, F. Ingenito, M. Marinelli, and G. Verona-Rinati, J. Instrum. 11, C12048 (2016).

27. M. Cipriani, F. Consoli, P. L. Andreoli, D. Batani, A. Bonasera, G. Boutoux, F. Burgy, G. Cristofari, R. De Angelis, G. Di Giorgio, J. E. Ducret, A. Flamigni, D. Giulietti,
K. Jakubowska, C. Verona, and G. Verona-Rinati, J. Instrum. 14, C01027 (2019).

28. J. Fuchs, P. Antici, E. d'Humières, E. Lefebvre, M. Borghesi, E. Brambrink, C. A. Cecchetti, M. Kaluza, V. Malka, M. Manclossi, S. Meyroneinc, P. Mora, J. Schreiber, T. Toncian, H. Pépin, and P. Audebert, Nature Phys. 2, 48 (2006).

29. P. L. Poole, L. Obst, G. E. Cochran, J. Metzkes, H. P Schlenvoigt, I. Prencipe, T. Kluge, T. Cowan, U. Schramm, D. W. Schumacher, and K. Zeil, New J. Phys. 20, 013019 (2018).

30. J. Schreiber, F. Bell, F. Grüner, U. Schramm, M. Geissler, M. Schnürer, S. Ter-Avetisyan, B. M. Hegelich, J. Cobble, E. Brambrink, J. Fuchs, P. Audebert, and D. Habs, Phys. Rev. Lett. 97, 045005 (2006).

31. K. Zeil, S. D. Kraft, S. Bock, M. Bussmann, T. E. Cowan, T. Kluge, J. Metzkes, T. Richter, R. Sauerbrey, and U. Schramm, New J. Phys. 12, 045015 (2010).

32. Y. Oishi, T. Nayuki, T. Fujii, Y. Takizawa, X. Wang, T. Yamazaki, K. Nemoto, T. Kayoiji, T. Sekiya, K. Horioka, Y. Okano, Y. Hironaka, K. G. Nakamura, K. Kondo, and A. A. Andreev, Phys. Plasmas 12, 073102 (2005).

33. C. M. Brenner, P. McKenna, and D. Neely, Plasma Phys. Control. Fusion 56, 084003 (2014). 